Received Date : 26-Jul-2016

Revised Date : 11-Jan-2017

Accepted Date : 11-Jan-2017

Article type : Research Report

\title{
Disruption of right Posterior Parietal Cortex by cTBS alters the control of body balance in quiet stance
}

David Kaulmann ${ }^{1}$, Joachim Hermsdörfer ${ }^{1}$, Leif Johannsen ${ }^{1,2}$

${ }^{1}$ Human Movement Science, Department of Sport and Health Sciences, Technische Universität München, Germany

${ }^{2}$ School of Health Sciences, Faculty of Medicine and Health Sciences, University of East Anglia, Norwich, United Kingdom

\section{Corresponding author:}

\section{Leif Johannsen}

School of Health Sciences,

Faculty of Medicine and Health Sciences,

University of East Anglia,

Norwich Research Park,

Norwich, NR4 7TJ, United Kingdom

Email: L.Johannsen@uea.ac.uk

Running title: Disruption of right Posterior Parietal Cortex by cTBS

Key words: cTBS, body sway control, light touch, posterior parietal cortex

This article has been accepted for publication and undergone full peer review but has not been through the copyediting, typesetting, pagination and proofreading process, which may lead to differences between this version and the Version of Record. Please cite this article as doi: $10.1111 /$ ejn. 13522

This article is protected by copyright. All rights reserved. 


\section{Abstract}

Control of body balance relies on the integration of multiple sensory modalities. Lightly touching an earth-fixed reference augments the control of body sway. We aimed to advance the understanding of cortical integration of an afferent signal from light fingertip contact (LT) for the stabilisation of standing body balance. Assuming that right-hemisphere Posterior Parietal Cortex (rPPC) is involved in the integration and processing of touch for postural control, we expected that disrupting rPPC would attenuate any effects of light touch. Eleven healthy right-handed young adults received continuous Theta Burst Stimulation over the left- and right-hemisphere PPC with sham stimulation as an additional control. Before and after stimulation, sway of the blindfolded participants was assessed in Tandem-Romberg stance with and without haptic contact. We analysed sway in terms of the variability of Centre-ofPressure (CoP) rate of change as well as Detrended Fluctuation Analysis of CoP position. Light touch decreased sway variability in both directions but showed direction-specific changes in its dynamic complexity: a positive increase in complexity in the mediolateral direction coincided with a reduction in the anteroposterior direction. rPPC disruption affected the control of body sway in two ways: first, it led to an overall decrease in sway variability irrespective of the presence of LT; second, it reduced the complexity of sway with LT at the contralateral, non-dominant hand. We speculate that rPPC is involved in the active exploration of the postural stability state, with utilization of LT for this purpose if available, by normally inhibiting mechanisms of postural stiffness regulation. 


\section{Introduction}

Keeping light contact ('light touch', LT) with objects in our environment augments the sensory feedback about the body's relative orientation in space and leads to reductions in body sway (Jeka \& Lackner, 1994). In order to integrate haptic information from the fingertips into the postural control loop, the central nervous system (CNS) may require interpretation of a local contact signal within the context of the body's overall proprioceptive state. This includes both arm posture and stance configuration, which could involve transformations of the haptic signal into an egocentric reference frame.

The posterior parietal cortices may be central components of a distributed network of neural circuits for the processing of somatosensory and proprioceptive information in ego-centric frames of reference (Longo et al., 2010; Medina \& Coslett, 2010; Bolton, 2015). For example, Azañón et al. (2010) showed that disruption of the right posterior parietal cortex (rPPC) impairs conscious position judgements of tactile stimuli on the left forearm relative to the face. With respect to the processing of haptic information for the control of body sway, Franzen and colleagues (2011) suggested that the postural control system has switched from a global to a local trunk-centred reference frame after light touch has been integrated into the postural control loop. Thus, right-hemisphere PPC (rPPC) seems like a good candidate to test for involvement in the processing of a fingertip signal within an egocentric reference frame for the control of body sway. 
Light touch of the dominant hand during quiet standing involves processing in the dominant left-hemisphere. Bolton et al. (2011) demonstrated that when the somatosensory feedback of the right hand contains sway-related information, brain activity at the left inferior parietal lobe caused by somatosensory-evoked potentials is modified by the specific postural context. In addition, Johannsen et al. (2015) investigated repetitive Transcranial Magnetic Stimulation (rTMS) over the left inferior parietal gyrus (IPG) to assess how stimulation affects the progression of sway before and after passive onset and removal of right-hand fingertip contact. They found that rTMS over the left IPG reduced overshoot of sway after contact removal, which indicates that this brain area may influence sensory reorganisation for sway control, for example in terms of directed tactile attention (Johannsen et al., 2015). There is evidence, however, that regions exist also in the non-dominant, right hemisphere for the processing of ipsilateral touch in the context of upright stance. Bolton et al. (2012) reported that disruption of the right prefrontal cortex alters the processing of right hand somatosensory-evoked potentials during contact with an earth-fixed reference.

Nevertheless. in the two stimulation studies reviewed above steady-state sway with light touch was not affected, which raises the question if disruption of another region such as the PPC changes the light touch effect during steady-state sway and if the rPPC in particular is contributing to the processing of touch irrespective of the haptically stimulated body side. The aim of this study was therefore to investigate the involvement of cortical processes represented within both posterior parietal cortices in the processing of haptic afferents for the control of balance. Assuming similar asymmetries between the hemispheres in terms of the 
processing of tactile input within spatial reference frames, as observed with respect to the distribution of spatial attention (Azanon et al., 2010) to the environment, we expected that disruption of the rPPC alters the integration of haptic afferences of both hands for sway control. In contrast, we expected that left-hemisphere PPC (IPPC) disruption would lead to an altered integration of touch of the contralateral hand only.

\section{Methods}

Participants

Eleven healthy right-handed young adults (mean age=25.45, SD 2.73; 6 women and 5 men) were recruited for the current study. Inclusion criteria were (1) right hand dominance, (2) no neurological or musculoskeletal disorders, (3) no balance impairment and (4) no reported cases of epilepsy. All participants were informed about the study protocol and signed a written informed consent. The study was approved by the Clinical Research Ethics committee of the Technical University of Munich.

\section{Procedure}

The experimental protocol was divided into three sessions. As a first session prior to the stimulation sessions a high resolution anatomical brain scan, consisting of a T1 MPRAGE (3T whole-body scanner, Signa HDx, GE Healthcare, Milwaukee, Wisconsin, USA) was carried out at the University Hospital Großhadern, Center for Sensorimotor Research. The brain scan was used in the following sessions for realtime neuronavigation in order to locate the respective stimulation area. 
Each TMS session consisted of a balance pre-test, the application of TMS and a balance post-test. The balance tests required blindfolded participants to stand on a force plate $(600 \mathrm{~Hz}$; Bertec FP4060-10, Columbus, Ohio, USA) in quiet TandemRomberg stance, while actively initiating and ceasing finger contact with an earthfixed referent in response to an acoustic signal. The earth-fixed contact reference point was placed in front of the participants. They held one arm slightly angled in front of the body and reaching straight forward. The other arm remained passive with the hand touching the stomach in order to prevent subjects from using arm movement to correct their body balance. Each balance testing consisted of 6 trials of at least 130 seconds (blocked, randomized order: 3 with the dominant hand, 3 with the non-dominant hand). Durations of the single trials varied due to the randomization of the length of the interval between contact events. TandemRomberg stance posture was adjusted according to the contacting hand. When the dominant hand contacted the reference point, the leg on the same side took the rear tandem position. When the contacting hand changed, so did the position of the feet. Participants were instructed to stand relaxed and not flex their knees to lock legs in position.

Each balance trial had six auditory triggered active transitions between Notouch and Touch (lowering the finger to the contact; "onset") and Touch and Notouch (raising the finger of the contact; "removal"). Every contact phase was at least 8 seconds long. Time points of contact onset and removal were randomized. We instructed participants to lightly press onto a contact plate downwards with a force around $1 \mathrm{~N}$. Before testing began, they practiced light touch in order to get a feeling for the applied force. Participants did not receive feedback about the contact force 
during a trial to avoid any attentional distractions and to prevent contacting from becoming an explicit precision task.

Body kinematics (4 Oqus 500 infrared cameras; 120 Hz; Qualisys, Göteborg, Sweden) and forces and torques at the reference contact location (6DoF Nano 17 force-torque transducer; $200 \mathrm{~Hz}$; ATI Industrial Automation, Apex, USA) were assessed. To capture body motion, reflective markers were placed at contacting fingertip, wrist, shoulders, C7, Sternum, hip and ankle.

During the TMS we applied continuous Theta Burst Stimulation (cTBS) of an intensity of $80 \%$ of the passive motor threshold for 60 seconds over the rPPC or IPPC (Fig. 1a; PMD70-pCool; MAG \& More, Munich, Germany). This protocol is widely used and stimulation effects can last from 20 minutes up to 1 hour (Staines \& Bolton, 2013). A staircase procedure was used to determine the passive motor threshold. In order to define the cTBS target areas, we used the MNI coordinates reported in Azañón et al. (2010), who stimulated the right-hemisphere human homologue of macaque ventral intraparietal area. We therefore expected that cTBS would disrupt activity in the Superior Parietal Lobule (SPL; Area 7A) and Intraparietal Sulcus (IPS) of the respective hemisphere. Stimulation locations were targeted using real-time neuronavigation software (TMS Neuronavigator; Brain Innovation, Maastricht, The Netherlands). During stimulation participants were seated comfortably on a reclined chair facing a wall and keeping their head straight. Participants needed five steps from the seat to the force plate. They had to cover this distance with their eyes closed in order to preserve any aftereffect of the stimulation as best as possible. 
Testing took place on two non-consecutive sessions with at least one day in between stimulation. The order of stimulation locations was randomized across participants with Sham stimulation being always the first stimulation in the second TMS-session. Sham stimulation was executed over the same target locations as for the cTBS (PMD70-pCool-Sham; MAG \& More, Munich, Germany). The location alternated across the sequence of participants, so that odd and even numbered participants received IPPC or rPPC sham stimulation respectively. Six participants received a IPPC/rPPC order and five a rPPC/IPPC order of stimulation.

\section{Data processing and statistical analysis}

The data of the force-torque transducer as well as the kinematic motion capture system were interpolated to $600 \mathrm{~Hz}$ and merged with the force plate data. Data were digitally low-pass filtered with a cut-off frequency of $10 \mathrm{~Hz}$ (dual-pass, 4th-order Butterworth). Center-of-Pressure (CoP) position was differentiated to yield rate of change parameters (dCoP) in order to remove low frequency drift. Based on the Normal force detected by the force-torque sensor, the onset and offset timepoints of each touching period was determined. In order to represent the time course of sway from $5 \mathrm{~s}$ before to $5 \mathrm{~s}$ after a contact event (onset/offset), the sway time series was segmented in to temporal bins of $500 \mathrm{~ms}$ duration. The standard deviation (SD) of anteroposterior $(\mathrm{AP})$ and mediolateral $(\mathrm{ML}) \mathrm{dCoP}$ was extracted for each bin. Data processing and extraction was conducted by MATLAB (MathWorks, 7.13 (2011b). Figure $1 \mathrm{~b}$ shows the progression of contact force and sway velocity over one trial. 
In order to characterize the fluctuation dynamics of body sway in nontransitory, steady postural states, segments of $5 \mathrm{~s}$ duration centered in between contact events were extracted from the time series of CoP position. These steadystate segments were appended in order to create time series of at least $25 \mathrm{~s}$ duration for Detrended Fluctuation Analysis (DFA) (Peng et al., 1995; Amoud et al., 2007; Duarte \& Sternad, 2008). We followed the basic algorithm as described by Peng et al. (1995) and obtained the DFA scaling exponent $\alpha$ as the slope of the linear regression of the log-log scaled detrended fluctuation plot as a function of a temporal window width of up to $10 \mathrm{~s}$ duration.

Sway in the anteroposterior and mediolateral directions and the scaling exponents were statistically analysed using 4-factorial repeated-measures ANOVA with (1) contacting hand (dominant vs. non-dominant hand; ipsilateral vs. contralateral hand relative to stimulation side), (2) location of stimulation (rPPC, IPPC and Sham), (3) effect of stimulation (Pre- and Post-cTBS) and (4) time course for onset and offset events (time bins) as within-subject factors. In order to test for steady-state effects, time bins $4.5 \mathrm{~s}$ to $3.5 \mathrm{~s}$ before the contact event and the three last extracted time bins (4s to $5 \mathrm{~s}$ ) after the contact event were contrasted for both each respective event type. For statistical significance a Greenhouse-Geisser corrected p-value of smaller 0.05 was used. A similar analysis was conducted for the derived contact force. All statistical analyses were carried out using SPSS (IBM SPSS Statistics 21). 


\section{Results}

Contacting force at the fingertip

Overall, average fingertip contacting force was $2.33 \mathrm{~N}$. Statistical analysis of the average contacting force and its variability did not reveal any effect of hand dominance, location of stimulation, effect of stimulation or any interactions between these factors.

\section{Variability of body sway during contact transitions}

Figure 2 shows the progression of sway variability over the time course of $5 \mathrm{~s}$ before a contact transition to $5 \mathrm{~s}$ after in bins of $500 \mathrm{~ms}$ duration before and after cTBS for each of the three stimulation locations. Before onset of fingertip contact, sway variability of the mediolateral direction is high and drops gradually to a lower level after contact is initiated $\left(F(19,190)=19.55, p<.001, \eta^{2}=.66\right)$. Sway variability remains low as long as contact is kept. Briefly after fingertip contact is removed, variability rises to higher, pre-contact levels $\left(F(19,190)=40.18, p<.001, \eta^{2}=.80\right)$. A similar progression of sway can be observed in the anteroposterior direction (onset $F(19,190)=16.83, p<.001, \eta^{2}=.63$; offset $\left.F(19,190)=16.91, p<.001, \eta^{2}=.63\right)$.

In terms of the general effect of touch, comparisons between the time bins from $4.5 \mathrm{~s}$ to $3.5 \mathrm{~s}$ before a contact event and the three last extracted time bins after the same contact event revealed a reduction in body sway variability with touch by $21 \%$ in the mediolateral direction (onset: $F(5,50)=36.96, p<.001, \eta^{2}=.79$; removal: $F(5,50)=122.49, p<.001, \eta^{2}=.93$ ) and by $22 \%$ in the anteroposterior direction (onset: $F(5,50)=56.12, p<.001, \eta^{2}=.85$; removal: $\left.F(5,50)=51.87, p<.001, \eta^{2}=.84\right)$. 
Regarding the effect of cTBS on sway variability, we found an interaction between stimulation location and stimulation effect in the mediolateral direction $\left(F(2,20)=6.12, p=.02, \eta^{2}=.38\right)$. We performed post-hoc ANOVAs for each stimulation location and found general sway reductions after cTBS for both the onset $\left(F(1,10)=5.14, p=.05, \eta^{2}=.34\right)$ and removal phases $\left(F(1,10)=5.28, p=.04, \eta^{2}=.35\right)$ after rPPC stimulation but after either IPPC or sham stimulation. In the mediolateral direction, stimulation over the rPPC decreased the sway variability in all phases with and without fingertip contact by $8 \%$. In contrast, sway variability was not reduced by IPPC $(3 \%$ increase) or sham stimulation $(1 \%$ increase). In the anteroposterior direction, a similar numerical trend could be observed (rPPC: $8 \%$ decrease; IPPC: $3 \%$ decrease; sham: $2 \%$ increase). However, the interaction between stimulation location and stimulation effect was not significant $\left(F(2,20)=1.78, p=.20, \eta^{2}=.15\right)$. Figure 3 shows sway variability averaged across all time bins (both onset and removal transitions combined) as a function stimulation location and effect for the mediolateral (Fig. 3a) and the anteroposterior direction (Fig. 3b).

\section{Sway fluctuation dynamics}

Detrended fluctuation analysis of sway for the mediolateral direction revealed that fingertip touch decreased the scaling exponent $\alpha$ in the DFA plots compared to Notouch (Fig. 4a; $\left.F(1,10)=18.91, p<.001, \eta^{2}=.65\right)$. In contrast, the scaling exponent $\alpha$ increased with touch in the anteroposterior direction $\left(F(1,10)=9.59, p=.01, \eta^{2}=.49\right)$. 
Furthermore, we found a marginally significant 4-way interaction between touch, hand, stimulation location and stimulation effect in the mediolateral direction $\left(F(2,20)=2.77, p=.10, \eta^{2}=.22\right)$. Post-hoc single comparisons expressed that $r P P C$ stimulation increased the scaling exponent $\alpha$ with contact of the non-dominant hand $\left(F(1,10)=6.06, p=.03, \eta^{2}=.38 ; F i g .5 b\right)$. In contrast, IPPC and sham stimulation resulted in no difference in this contact condition (Fig. 5a and Fig. 5c).

\section{Discussion}

We aimed evaluate the effects of disruption by cTBS of the PPC in both hemispheres on the processing of fingertip light touch for body sway control in Tandem Romberg stance. Surprisingly, after stimulation of the rPPC, the general level of sway variability was decreased. This encompassed all trial phases including those in which light fingertip contact was applied and body sway reduced by the augmented sensory feedback. Light touch changed the sway dynamics in a direction-specific manner in favour of the mediolateral direction. In the mediolateral direction, however, a second effect of rPPC disruption became visible. After the stimulation, the sway dynamics degraded in those phases in which light contact was kept with the non-dominant, contralateral hand.

The general reduction after rPPC disruption appears like an unexpected improvement in sway. Reduced sway variability, however, does not necessarily mean that individuals possess a greater degree of stability in terms of the ability to compensate a balance disturbance. For example, variability is adjusted by the postural control system according to the demands of a specific supra-postural task and seems to be necessary for flexible reactions to external perturbations (Balasubramaniam et al., 2000). It can be argued that the reduction in sway reflects 
an unfavorable effect in terms of participants becoming less adaptive and less able to compensate for unexpected perturbations (Lipsitz, 2002) after rPPC disruption. Possibly, rPPC disruption resulted in an increase in overall postural stiffness by muscular co-contractions and therefore showed reduced body sway variability (Maurer \& Peterka, 2005).

If disruption of the rPPC results in increased stiffness, then the question remains which functional aspect of body sway control the rPPC does represent? We like to propose a functional equilibrium between a process that controls body stiffness and a process that actively explores the own body's current state of stability in the context of the specific postural configuration and orientation (Riccio et al., 1992). Control of stiffness plays a crucial part when interacting with the environment, for example to gain postural support or when anticipating external perturbations. In the absence of an external perturbation, active stability state exploration would probe for any deviation from the body's equilibrium point by registering the forces and torques required to counteract any environmental dynamics exerted onto the body. Possibly, the rPPC is involved in this active exploration process.

Yadav and Sainburg (2014) propose a distinction between two neural systems for limb control, one for predictive control of arm movements and the other for control of arm stiffness (impedance). The former system is attributed to the dominant (left) hemisphere in right-dominant participants, while the latter to the non-dominant (right) hemisphere (Yadav \& Sainburg, 2014). Several studies in stroke patients have implied that the right hemisphere may dominate the control of body sway (Rode et al., 1997; Peurala et al., 2007; Tasseel-Ponche et al., 2015). Assuming that stiffness control by the right hemisphere generalizes from the non-dominant arm to the control of body sway, our results suggest that stiffness control and active exploration are two 
processes coordinated within the right hemisphere. If the rPPC contributes to active exploration, the question remains, which right-hemisphere regions control stiffness. It is likely that the rPPC is part of a network, which is distributed across several brain regions responsible for maintaining a functional equilibrium (Bolton, 2015). Studies reveal a wide spread of different cortical areas involved in the control of balance ranging from the prefrontal cortex, primary motor cortex and the parietal cortex (Mihara et al. 2012) to the basal ganglia (Visser \& Bloem, 2005). Functions of the basal ganglia include muscle tone regulation and control of automatic postural responses and patients with dysfunction in that area often show axial stiffness, gait freezing or co-contraction (Visser \& Bloem, 2005). Thus, the basal ganglia seem like a good candidate to be involved in stiffness or impedance control. The prefrontal, primary motor and parietal cortices might form the exploratory processes for balance control.

Our results show reduced variability of sway with light touch in both directions. Although apparently a similar effect occurred in both directions, there might be differences between mediolateral and anteroposterior sway as the complexity measure of sway dynamics showed opposite changes for both directions. While the scaling exponent $\alpha$ decreases with light touch in the mediolateral direction, it rises in the anteroposterior direction (Fig. 4). In both directions the scaling exponent $\alpha$ was greater than 1, which is interpreted as a non-stationary signal with low long term selfsimilarity and reduced complexity. $1 / f$ noise $(\alpha \sim 1)$ is associated with a high complexity and is present in many natural, healthy, unperturbed systems (Duarte \& Zatsiorsky, 2001). Deviations from this complexity range might result in pathophysiological disturbances (Duarte \& Zatsiorsky, 2001; Hausdorff et al., 1995). Perhaps, the generally greater than 1 scaling exponent $\alpha$ in our study is an 
expression of the increased postural challenge caused by the stance position with eyes closed. Although the scaling exponent $\alpha$ does not decrease to a value close to or below 1 , a reduction could be observed in the mediolateral direction at the cost of an increase in the anteroposterior direction with light touch.

It might be possible that with light contact the dynamics of sway became more direction-specific. Participants stood in Tandem-Romberg stance, which introduces imbalance especially in the mediolateral direction. Therefore, this direction might have become more task-goal relevant in terms of the utilization of the haptic signal for the control of sway. These effects in the mediolateral direction occurred despite the contact point being orientated along the orthogonal, anteroposterior direction. Effects might be even stronger if the contact point is positioned along the mediolateral axis (Jeka et al., 1998). We placed the contact point on the midline to enable quick switching between the two hands as two force-torque sensors were not available to us for placement of one contact point on each side. The sway dynamics do not show a general effect of rPPC disruption. Instead, results show an increase in the scaling exponent $\alpha$ after disruption of the rPPC with fingertip contact of the nondominant, contralateral hand. It might be that the disruption led to a non-optimal integration of haptic information for body sway control. Ishigaki et al. (2016) demonstrated that processing of a haptic signal when it contains information about body sway relative to an earth-fixed reference reduces cortical activity in the contralateral left-hemisphere parietal lobe as determined by EEG. Unfortunately, they did not assess the effect of contact with the non-dominant (left) hand. We would expect similar contralateral activity reductions in the right-hemisphere parietal lobe. 
We did not find an increase of the scaling exponent $\alpha$ in the dominant hand after IPPC disruption. It might simply be that we missed the adequate target location in the left-hemisphere parietal lobe to induce any disruptive effects. It might also be possible, however, that differences between the hemispheres exist with respect to the processing of tactile feedback for sway control. In a previous study, we did not find any disruptive effects of rTMS over the left IPG and left middle frontal gyrus on steady-state body sway with LT (Johannsen et al., 2015). It may be that a disruption of the left-hemisphere was compensated by other brain regions for example the rPPC.

Figure 6 summarizes a simple functional model of interhemispheric interaction, which could underlie our effect patterns. Assuming that rPPC is part of a neural architecture which controls active exploration of the postural stability state opposed by other structures which regulate postural stiffness, rPPC might utilize the haptic signal at the fingertips for this task. rPPC may be disposed to processes haptic information in ego-centric reference frames (Longo et al., 2010; Medina \& Coslett, 2010) from both hands, while IPPC processesand relays haptic information from the contralateral hand only (Fig. 6a). If rPPC is disrupted by cTBS, active stability state exploration may be impaired leading to reduced body sway (Fig. 6b). In addition, the utilization of haptic information for sway control from both hands may be affected. In terms of the sway dynamics, a deficit becomes apparent for the contralateral (relative to rPPC), non-dominant hand as the left hemisphere can still process and relay in a signal from the contralateral (relative to IPPC), dominant hand. Finally, if IPPC is disrupted by cTBS (Fig. 6c), only processing of the dominant hand's haptic information is impaired, which can be compensated by rPPC's own access to ipsilateral haptic information. For example, Borchers et al. (2011) reported 
a stroke patient, who demonstrated a proprioceptive deficit for both hands after a right postcentral lesion. Ishigaki et al. (2016), however, did not report bilateral activity changes during quiet stance with light touch but exclusively in the dominant hemisphere contralateral to the contacting hand. As both hemispheres were undisturbed physiologically in their experiment, it may be that any ipsilateral activity changes in the right hemisphere were suppressed.

Continuous TBS over the right or left PPC had no effect on the applied finger force and its variability. Even though average contacting force exceeded $1 \mathrm{~N}$, we still consider it a light touch since the applied forces were still not sufficient to provide mechanical support. Moreover, we argue that the light touch in our experiment is a more natural evolving light touch as we tried to avoid turning it into an explicit precision task by including online force feedback. It might be possible, however, that the applied touch in our experiment is processed differently than light touch of lesser than $1 \mathrm{~N}$. Jeka and Lackner (1994) reported that feedback delays between fingertip forces and postural adjustments were much longer and the coupling weaker -with contact below $1 \mathrm{~N}$ compared to contact with unconstrained forces showing shorter time lags and stronger coupling between fingertip forces and postural adjustments. In this respect the latter might resemble classical supraspinal, long-latency reflexes. Average contact forces in the unconstrained condition in Jeka and Lackner (1994b), however, exceeded $4 \mathrm{~N}$, which is at least twice the amount of contact forces in our present study. Whether the processing of haptic feedback below $1 \mathrm{~N}$ or above $4 \mathrm{~N}$ is linked with a continuous functional gradient or whether a discontinuity exists between these two ranges is unknown to date and worth further investigation. As contact forces in our present study are closer to the $1 \mathrm{~N}$ range, we 
suggest that the haptic signals in our study should still be considered 'light' but we cannot exclude the possibility that this was the reason disruption of the PPC led to no changes in the level of sway specifically with light touch.

In conclusion, we replicated the traditional effect of light touch on body with decreased sway variability but showed direction-specific changes in its complexity. Moreover, we showed that overall sway variability decreases, in addition to the light touch effect, while the sway complexity increases when utilizing haptic information from the non-dominant, contralateral hand after rPPC disruption. We speculate that an increase in postural stiffness could result from lowered inhibition of stiffness regulation by a disrupted process, which is engaged in actively exploring the body's stability state. We propose a simple functional model of interhemispheric interactions, which could explain our results pattern by the assumption of an asymmetry between the $\mathrm{rPPC}$ and IPPC regarding bilateral utilization of haptic information for the control of body sway.

\section{Acknowledgements}

We like to thank Dr. Thomas Stephan and Dr. Virginia Flanagin for their help with collecting the anatomical brain scans. We acknowledge the financial support by the Federal Ministry of Education and Research of Germany (BMBF; 01EO1401) and by the Deutsche Forschungsgemeinschaft (DFG) through the TUM International Graduate School of Science and Engineering (IGSSE). 


\section{Abbreviations:}

AP: Anteroposterior

CoP: Center-of-Pressure

DFA: Detrended Fluctuation Analysis

IPG: Inferior Parietal Gyrus

ML: Mediolateral

TBS: Theta Burst Stimulation

TMS: Transcranial Magnetic Stimulation

PPC: Posterior Parietal Cortex

SD: Standard Deviation

\section{Competing interests}

The authors declare no conflict of interest.

\section{Author contributions}

DK and LJ contributed equally to the study design, data collection, data analysis and manuscript preparation. $\mathrm{JH}$ contributed to study design and manuscript preparation.

\section{Data accessibility}

The experimental data will be accessible via the institutional media repository of the Technical University Munich (https://mediatum.ub.tum.de).

\section{References}

Amoud, H., Abadi, M., Hewson, D.J., Michel-Pellegrino, V., Doussot, M. \& Duchêne, J. (2007) Fractal time series analysis of postural stability in elderly and control subjects. Journal of NeuroEngineering and Rehabilitation, 4, 1-12. 
Azanon, E., Longo, M.R., Soto-Faraco, S. \& Haggard, P. (2010) The posterior parietal cortex remaps touch into external space. Current biology : CB, 20, 1304-1309.

Balasubramaniam, R., Riley, M.A., Turvey, M.T. (2000) Specificity of postural sway to the demands of a precision task. Gait and Posture, 11, 12-24.

Bolton, D.A. (2015) The role of the cerebral cortex in postural responses to externally induced perturbations. Neuroscience and biobehavioral reviews, 57, 142-155.

Bolton, D.A., Brown, K.E., Mcllroy, W.E. \& Staines, W.R. (2012) Transient inhibition of the dorsolateral prefrontal cortex disrupts somatosensory modulation during standing balance as measured by electroencephalography. Neuroreport, 23, 369-372.

Bolton, D.A., Mcllroy, W.E. \& Staines, W.R. (2011) The impact of light fingertip touch on haptic cortical processing during a standing balance task. Exp. Brain Res, 212, 279291.

Borchers, S., Hauser, T.K. \& Himmelbach, M. (2011) Bilateral hand representations in human primary proprioceptive areas. Neuropsychologia, 49, 3383-3391.

Duarte, M. \& Sternad, D. (2008) Complexity of human postural control in young and older adults during prolonged standing. Experimental brain research, 191, 265-276.

Franzén, E., Gurfinkel, V.S., Wright, W.G., Cordo, P.J. \& Horak, F.B. (2011) Haptic touch reduces sway by increasing axial tone. Neuroscience, 174, 216-223.

Ishigaki, T., Ueta, K., Imai, R. \& Morioka, S. (2016) EEG frequency analysis of cortical brain activities induced by effect of light touch. Experimental brain research, 234, 14291440.

Jeka, J.J. \& Lackner, J.R. (1994) Fingertip contact influences human postural control. Experimental brain research, 100, 495-502.

Jeka, J.J., Ribeiro, P., Oie, K. \& Lackner, J.R. (1998) The structure of somatosensory information for human postural control. Motor Control, 2, 13-33.

Johannsen, L., Hirschauer, F., Stadler, W. \& Hermsdorfer, J. (2015) Disruption of contralateral inferior parietal cortex by $1 \mathrm{~Hz}$ repetitive TMS modulates body sway following unpredictable removal of sway-related fingertip feedback. Neuroscience letters, 586, 13-18.

This article is protected by copyright. All rights reserved. 
Lipsitz, L.A. (2002) Dynamics of stability the physiologic basis of functional health and frailty. The Journals of Gerontology Series A: Biological Sciences and Medical Sciences, 57, B115-B125.

Longo, M.R., Azanon, E. \& Haggard, P. (2010) More than skin deep: body representation beyond primary somatosensory cortex. Neuropsychologia, 48, 655-668.

Maurer, C. \& Peterka, R.J. (2005) A new interpretation of spontaneous sway measures based on a simple model of human postural control. Journal of neurophysiology, 93, 189-200.

Medina, J. \& Coslett, H.B. (2010) From maps to form to space: touch and the body schema. Neuropsychologia, 48, 645-654.

Peng, C.K., Havlin, S., Stanley, H.E. \& Goldberger, A.L. (1995) Quantification of scaling exponents and crossover phenomena in nonstationary heartbeat time series. Chaos, 5, 82-87.

Peurala, S.H., Kononen, P., Pitkanen, K., Sivenius, J. \& Tarkka, I.M. (2007) Postural instability in patients with chronic stroke. Restorative neurology and neuroscience, 25, 101-108.

Riccio, G.E., Martin, E.J. \& Stoffregen, T.A. (1992) The role of balance dynamics in the active perception of orientation. Journal of Experimental Psychology: Human Perception and Performance, 18, 624.

Rode, G., Tiliket, C. \& Boisson, D. (1997) Predominance of postural imbalance in left hemiparetic patients. Scandinavian journal of rehabilitation medicine, 29, 11-16.

Staines, W.R. \& Bolton, D.A. (2013) Transcranial magnetic stimulation techniques to study the somatosensory system: research applications. Handbook of Clinical Neurology, 116, 671-679.

Tasseel-Ponche, S., Yelnik, A.P. \& Bonan, I.V. (2015) Motor strategies of postural control after hemispheric stroke. Clinical neurophysiology, 45, 327-333.

Yadav, V. \& Sainburg, R.L. (2014) Limb dominance results from asymmetries in predictive and impedance control mechanisms. PLoS one, 9, e93892.

This article is protected by copyright. All rights reserved. 


\section{Figure legends}

Figure 1. (A) An illustration of real-time neuronavigation for a participant. Black circles mark the stimulation location in the left and right PPC. (B) A sample trial for single participant. Normal contact force and mediolateral CoP rate of change are plotted across the time course of $140 \mathrm{~s}$ trial. (C) Generic overview of the two stimulation sessions.

Figure 2. The time course of mediolateral sway across 20 bins of $500 \mathrm{~ms}$ width at contact onset and removal. The black lines indicate body sway variability before cTBS and the dashed lines following cTBS. Error bars indicate standard error of the mean. PPC: posterior parietal cortex.

Figure 3. Grand averaged body sway variability as a function of stimulation location before (light grey points) and after (dark grey points) cTBS for the mediolateral (A) and anteroposterior direction (B). Horizontal bars indicating the mean value averaged across all participants. ${ }^{*}: p<0.05 .+: p<.10$. IPPC: left posterior parietal cortex. rPPC: right posterior parietal cortex.

Figure 4. Scaling exponent as a function of light touch contact for the mediolateral and anteroposterior direction. Horizontal bars indicating the mean value averaged across all participants. *: $p<0.05$.

Figure 5. Scaling exponent as a function of touch contact with the dominant and nondominant hand before (black points) and after (light grey points) cTBS for (A) Left PPC stimulation, (B) Right PPC stimulation and (C) Sham stimulation. Horizontal 
bars indicating the mean value averaged across all participants. *: $p<0.05$. IPPC: left posterior parietal cortex. rPPC: right posterior parietal cortex.

Figure 6. A simplistic functional model of interhemispheric interactions for active stability state exploration. (A) No cTBS disruption. (B) cTBS over the right parietal cortex. (C) cTBS over the left parietal cortex. IPPC: left posterior parietal cortex. rPPC: right posterior parietal cortex. Lightning symbol: cTBS disruption. $\mathrm{X}$ : dysfunction. 
A

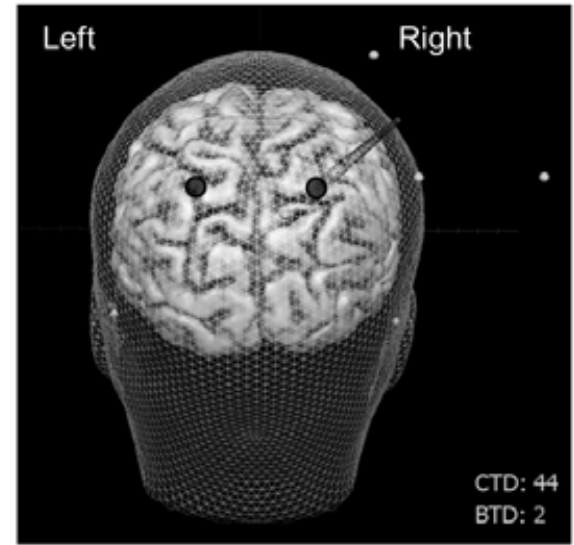

B

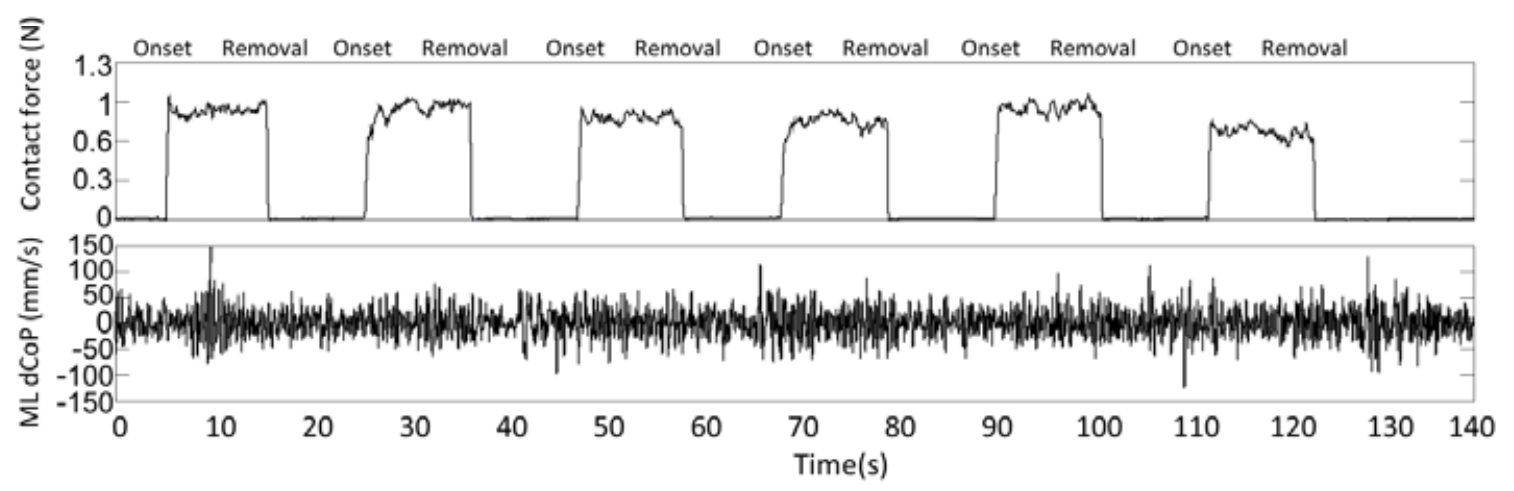

C

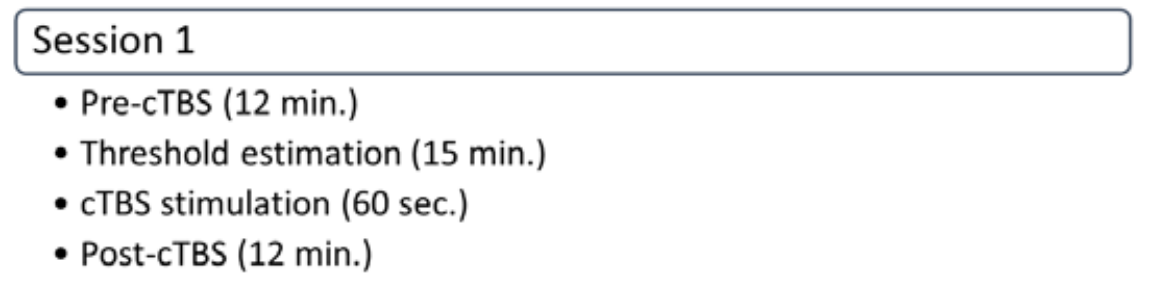

\section{Session 2}

- Pre-Sham (12 min.)

- Sham stimulation (60 sec.)

- Post-Sham/Pre-cTBS (20 min.)

- cTBS stimulation (60 sec.)

- Post-cTBS (12 min.)

Figure 1.

This article is protected by copyright. All rights reserved. 


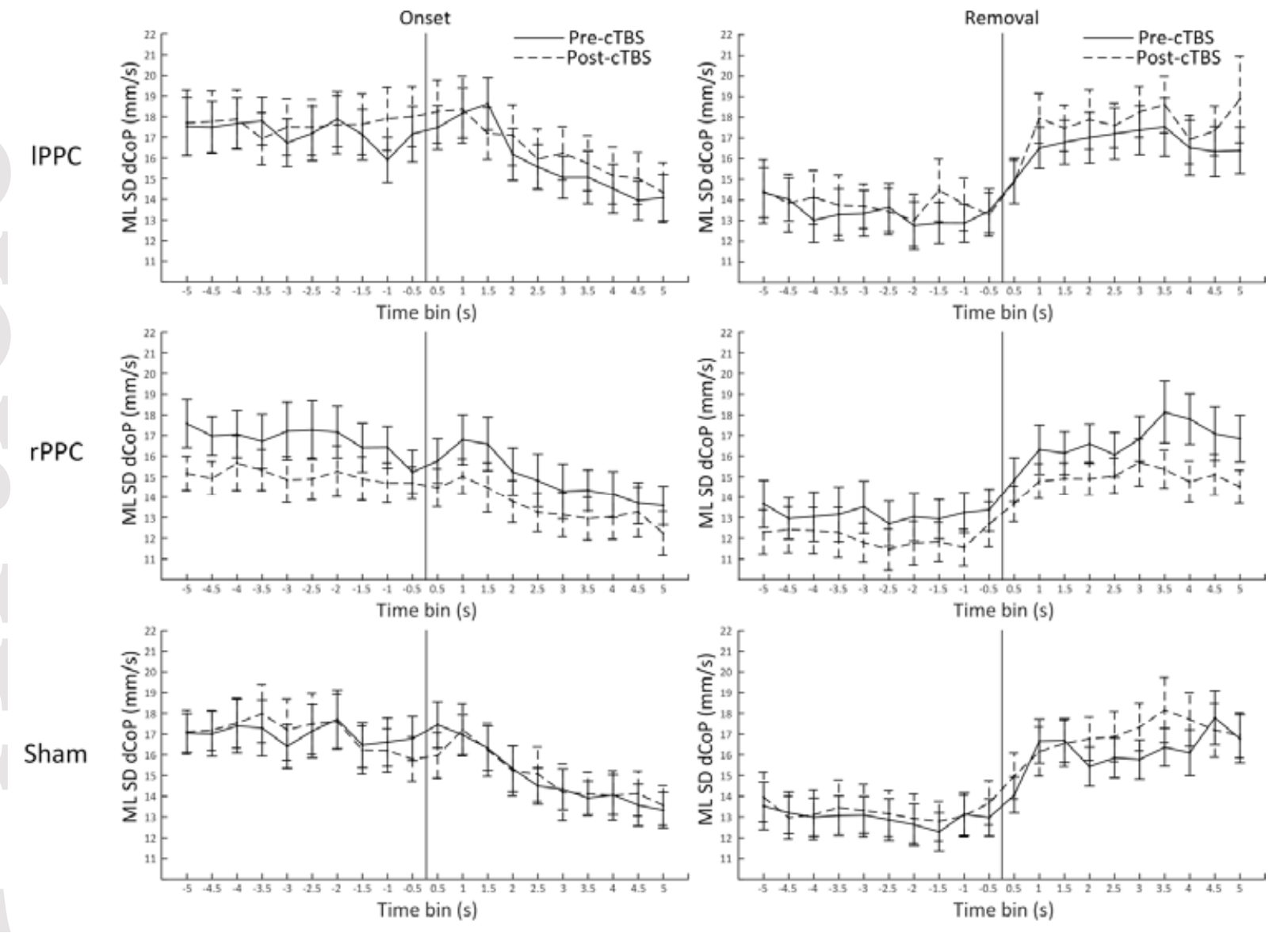

Figure 2.

This article is protected by copyright. All rights reserved. 
A

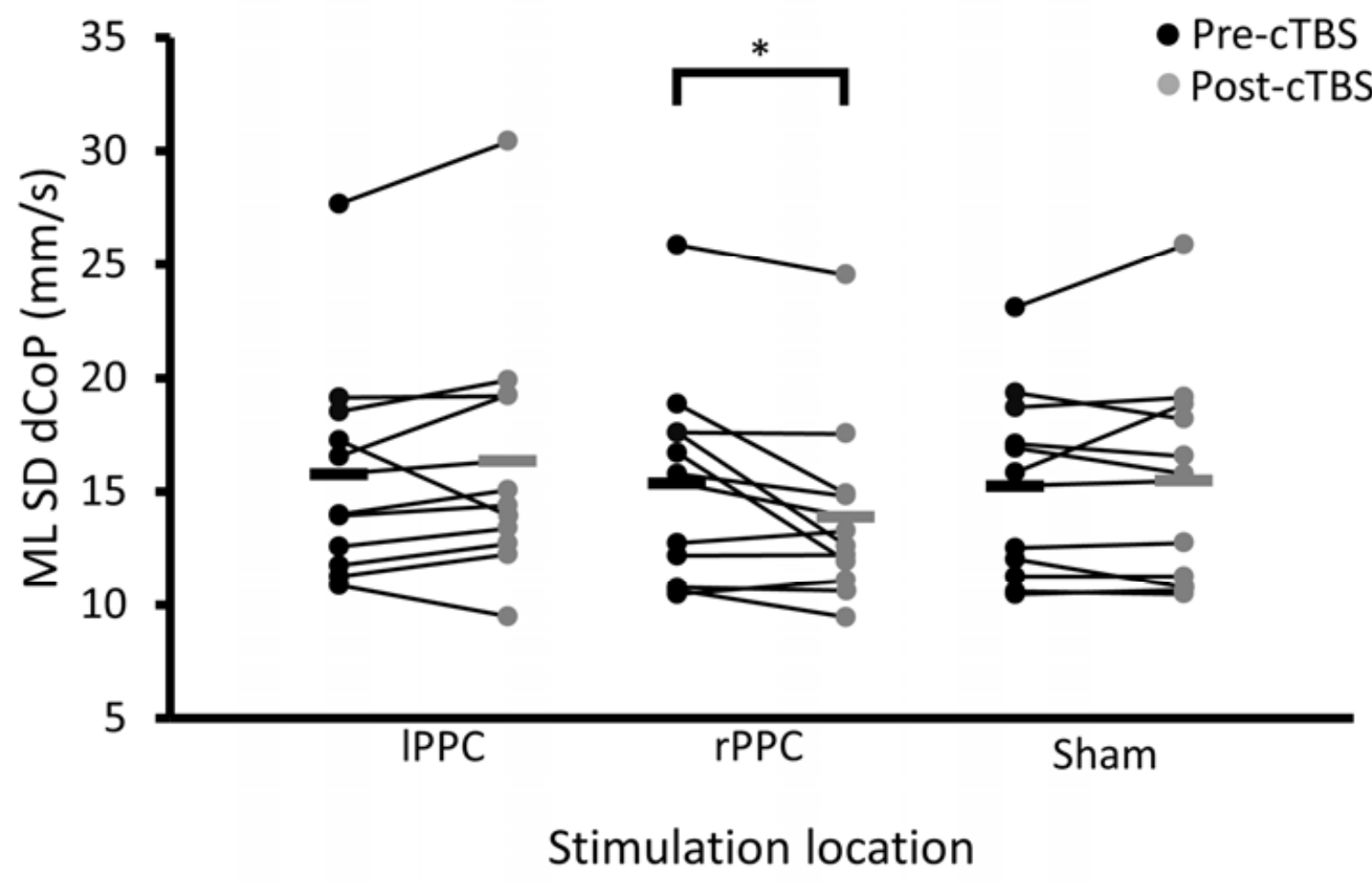

B

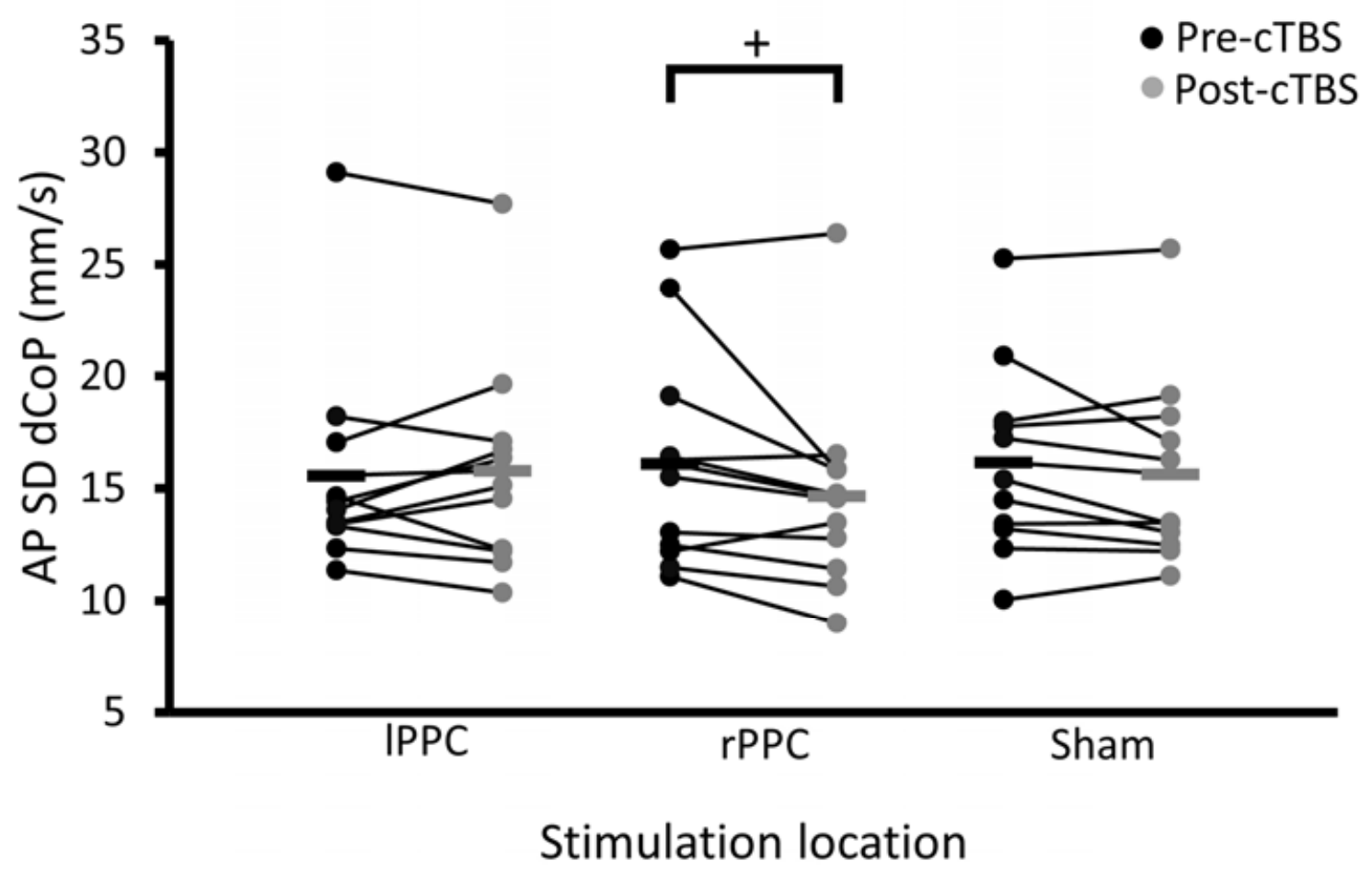

Figure 3. 


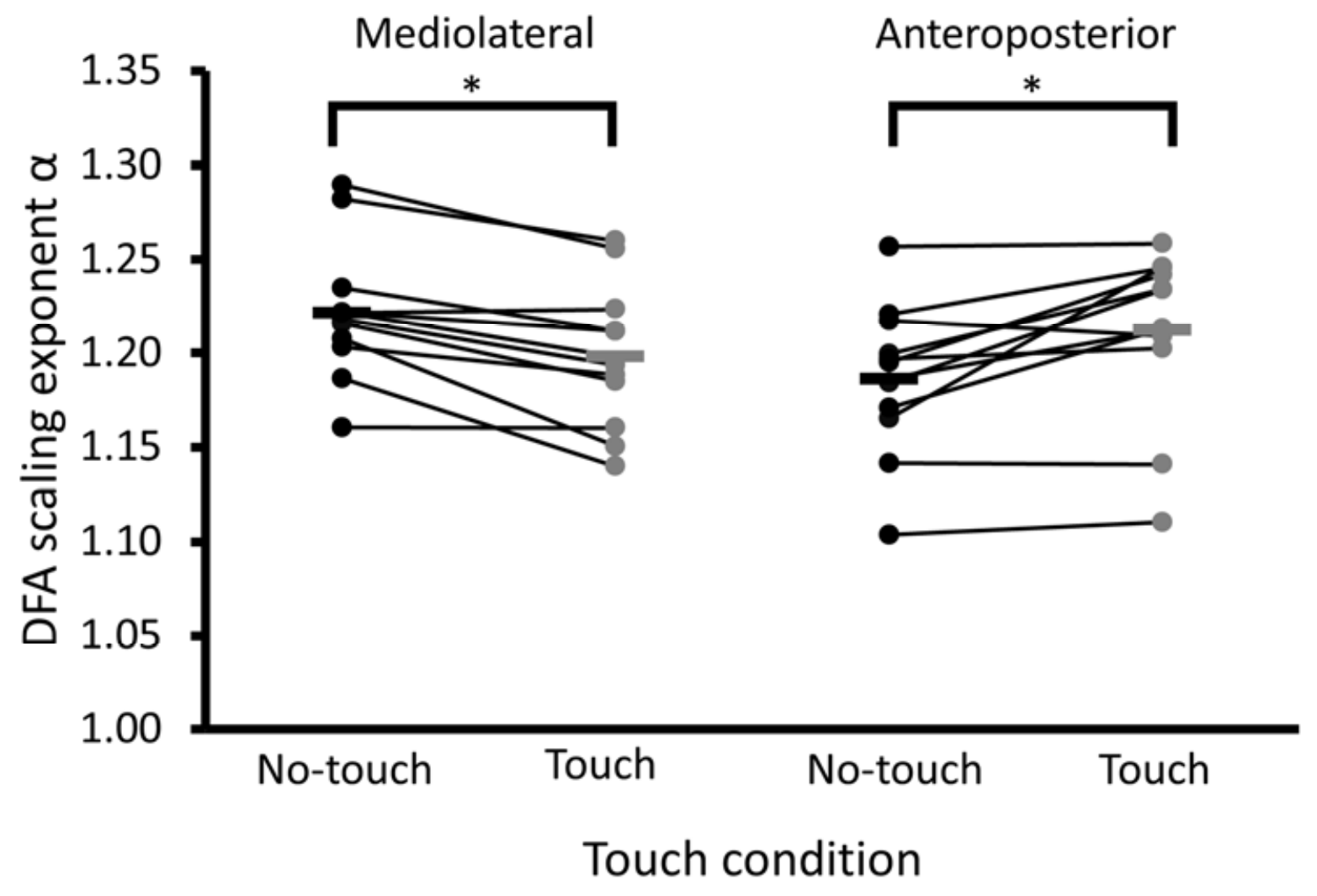

Figure 4. 
A

IPPC

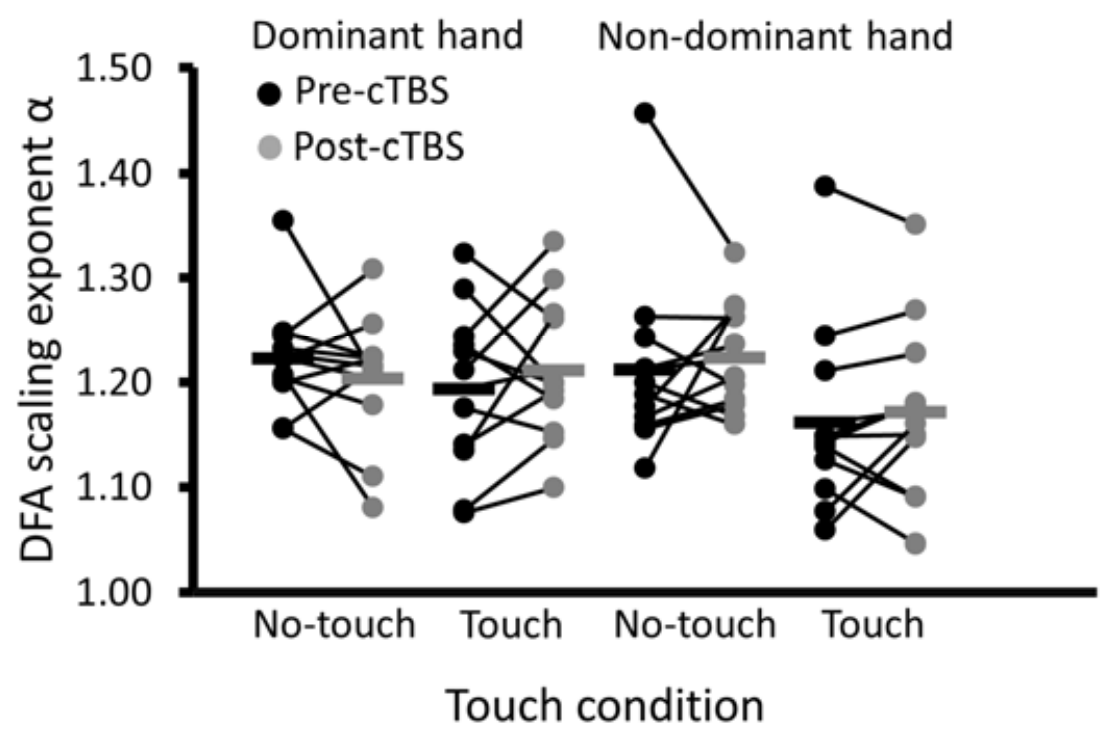

B

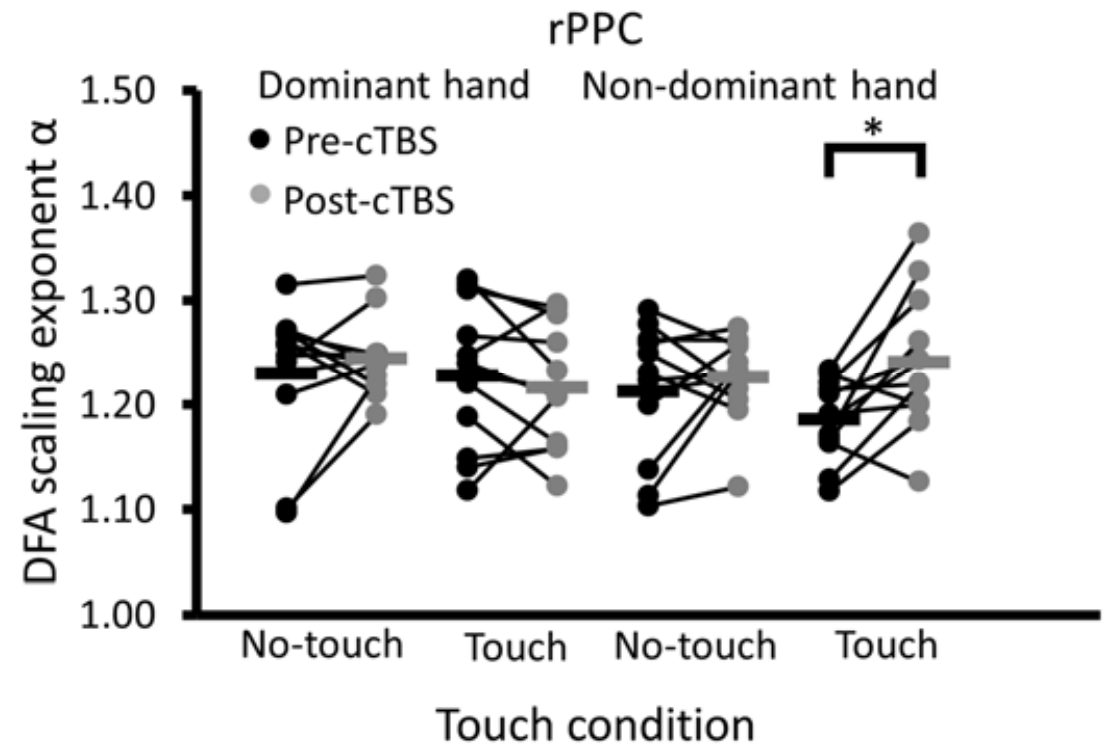

C

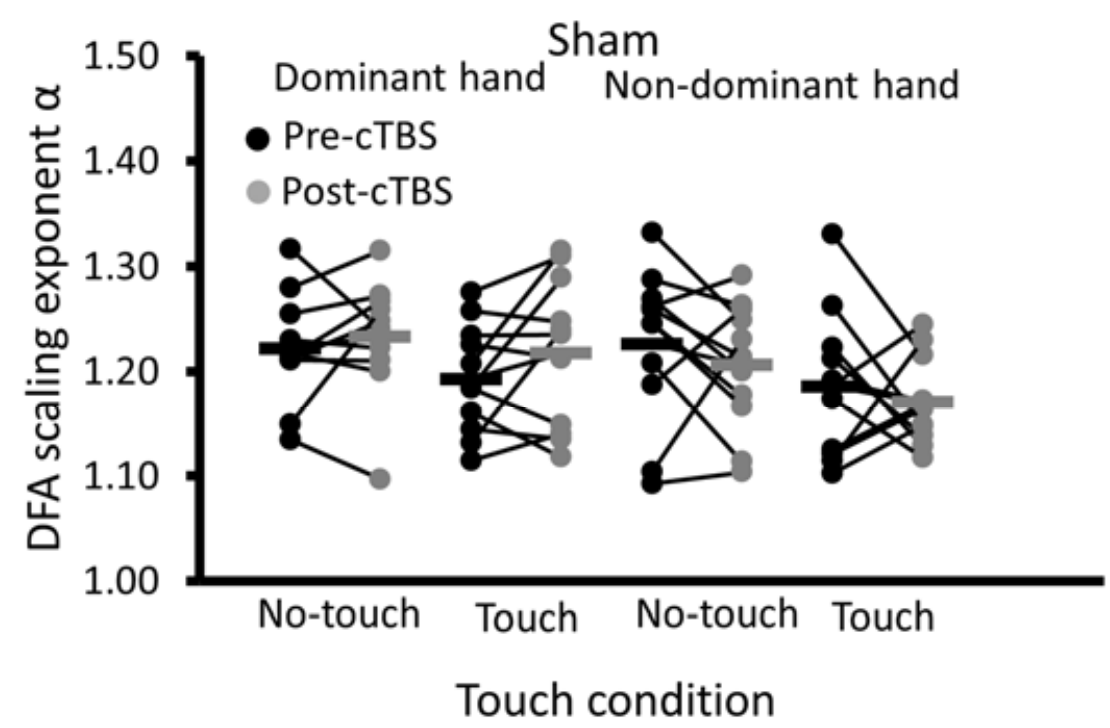

Figure 5.

This article is protected by copyright. All rights reserved. 
A

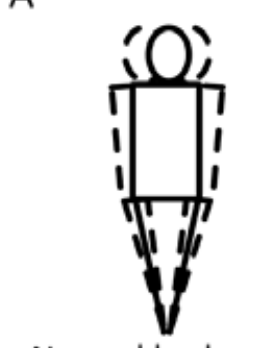

Normal body sway

B

C
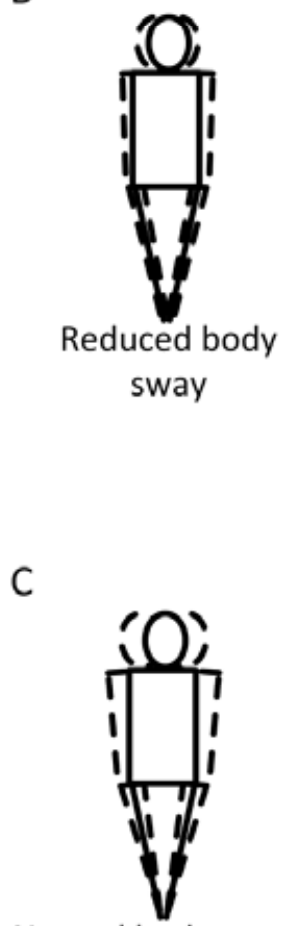

Normal body sway
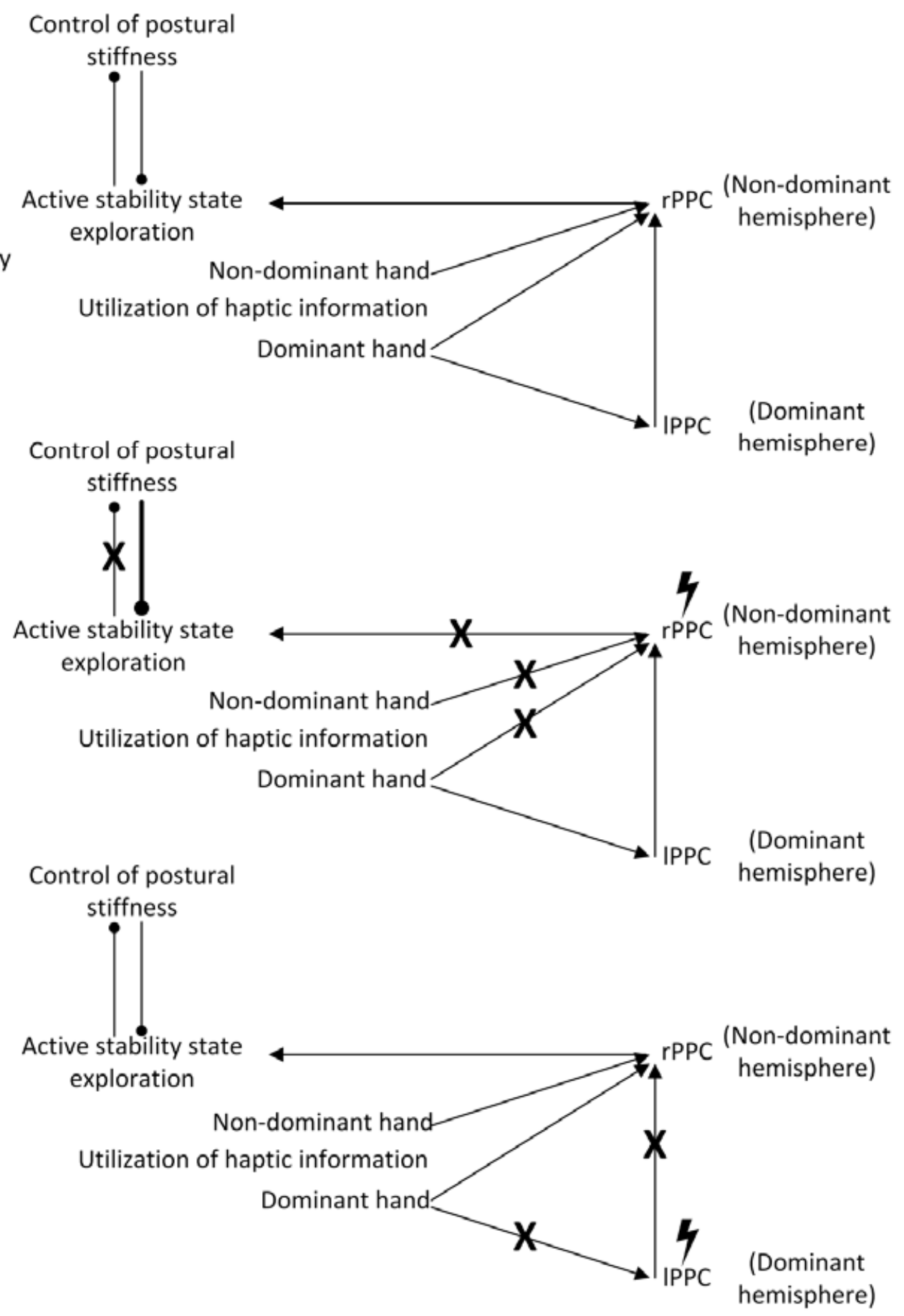

Figure 6.

This article is protected by copyright. All rights reserved. 